\title{
Cross-national comparison of the link between socioeconomic status and emotional and behavioral problems in youths
}

\author{
Floor V. A. van Oort · Jan van der Ende • \\ Martha E. Wadsworth • Frank C. Verhulst • \\ Thomas M. Achenbach
}

Received: 8 April 2009/Accepted: 21 January 2010/Published online: 18 February 2010

(C) The Author(s) 2010. This article is published with open access at Springerlink.com

\begin{abstract}
Introduction In previous longitudinal studies in the US, lower socioeconomic status (SES) was associated with more emotional and behavioral problems. It remains unclear whether these findings can be generalized outside the US, as different countries vary in their health care systems and prevention of psychopathology in youth. Therefore, we studied the same associations in a comparable sample in The Netherlands and directly tested for differences between the US and The Netherlands.

Methods The US $(N=833)$ and Dutch $(N=708)$ population samples were followed-up for 9 years. Age at baseline ranged from 8 to 16 years. Parents filled out behavior checklists.

Results Analyses revealed very few differences between the two countries. In both countries, SES predicted syndrome scores and cumulative prevalence rates for internalizing and externalizing problems (withdrawn and aggressive behavior) and for thought and attention Problems. The SES gradient in syndrome scores was stable over time. Only for withdrawn behavior, the gradient was larger in young adulthood.

Conclusion Although the health care systems differ between the US and The Netherlands, the socioeconomic disparities in emotional and behavioral problems were similar.
\end{abstract}

F. V. A. van Oort $(\bowtie)$. J. van der Ende · F. C. Verhulst Department of Child and Adolescent Psychiatry, Erasmus MC, P.O. Box 2060, 3000 CB Rotterdam, The Netherlands e-mail: f.vanoort@erasmusmc.nl

M. E. Wadsworth

Department of Psychology, University of Denver, Denver, USA

T. M. Achenbach

Department of Psychiatry, University of Vermont,

Vermont, USA
Keywords Socioeconomic · Psychopathology · Youths $\cdot$ Prospective $\cdot$ Cross-national

\section{Introduction}

Previous studies have shown that rates of psychopathology are higher among individuals with lower socioeconomic status (SES) than those with higher socioeconomic status (e.g., [9, 14, 16]. Fewer studies have reported on these associations in children and adolescents [18]. The main hypothesis for the association is the social causation hypothesis, which posits that people with low SES develop psychological problems because of living with adversity, and/or through less access to effective treatment. A second, complementary, hypothesis is the social selection hypothesis that posits that individuals with psychological problems drift down the SES ladder because of their psychopathology and their resultant inability to fulfill expected role obligations. Both hypotheses predict differential incidence rates and differential cumulative prevalence rates by SES. The degree to which each hypothesis accounts for variation in a particular disorder has important implications for the treatment and prevention of that disorder.

Longitudinal analyses of children's problems provide a purer test of social causation effects than is possible with adults, as children's behaviors do not determine a family's SES. In a longitudinal study in the US, Wadsworth and Achenbach [25] reported differential incidence by SES for elevated scores on syndromes of anxious/depressed behavior, somatic complaints, thought problems, delinquent behavior, and aggressive behavior. SES-linked differential cumulative prevalence was found for elevated scores on syndromes of thought problems, attention problems, delinquent behavior, and aggressive behavior. It is 
not yet clear whether these findings can be generalized outside the US, as countries vary in their health care systems and prevention of psychopathology in youth [15]. We therefore assessed socioeconomic differences in a prospective community sample in The Netherlands and directly tested for differences between the US and The Netherlands. In The Netherlands, access to health care is free for children, whereas access to health care in the US is determined by differences in income level [5]. Furthermore, monitoring and identification of mental health problems in primary and secondary schools are tasks of the youth healthcare in the Netherlands, but not in the US. The coverage is high in schools in low as well as high socioeconomic neighborhoods. Consequently, weaker associations with SES might be expected in The Netherlands than in the US.

\section{Method}

Samples

\section{US sample}

The US national sample has been described in detail by Achenbach et al. [3]. The original 1986 sample consisted of 2,734 in the age of 4- to 16-year-old children. Reassessments were performed in 1989, 1992, and 1995. The sample was selected to be representative of the 48 contiguous states with respect to ethnicity, socioeconomic status, geographic region, and urbanicity. The baseline (1986), 3-year (1989), and 9-year (1995) assessments were used for the current analyses. To be comparable with the Dutch sample, we selected only non-Hispanic Whites aged $8-16$ years at baseline with complete data $(N=833)$. At baseline and 3-year assessments, parents were interviewed regarding mental health symptoms and socioeconomic status. The 9-year assessment obtained parents' reports from mailed questionnaires. Table 1 displays the demographic characteristics of the sample. Sample attrition was not selective for problem scores, gender, or age, but dropout rates were slightly elevated for lower SES participants [3].

\section{Dutch sample}

The Dutch sample originally consisted of 4- to 16-year-old children who were randomly drawn from the Dutch province of Zuid-Holland in 1983 [23]. The children were reassessed in 1985, 1987, 1989, 1991, and 1997. Participating parents were interviewed regarding mental health symptoms and socioeconomic status. We used data from the 1987, 1989, 1991, and 1997 re-assessments. To provide
Table 1 Demographic characteristics of the samples

\begin{tabular}{|c|c|c|c|c|c|c|c|}
\hline \multirow[t]{2}{*}{ Characteristic } & \multicolumn{2}{|l|}{ US } & \multicolumn{2}{|c|}{ Netherlands } & \multicolumn{2}{|l|}{ Total } & \multirow[t]{2}{*}{$P^{\mathrm{b}}$} \\
\hline & $N$ & $\%$ & $N$ & $\%$ & $N$ & $\%$ & \\
\hline$N$ & 833 & & 708 & & 1,541 & & \\
\hline SES & & & & & & & 0.001 \\
\hline Low & 243 & 29 & 215 & 30 & 458 & 30 & \\
\hline Medium & 382 & 46 & 370 & 52 & 752 & 49 & \\
\hline High & 208 & 25 & 123 & 17 & 331 & 21 & \\
\hline Gender & & & & & & & 1.00 \\
\hline Male & 387 & 46 & 329 & 46 & 716 & 46 & \\
\hline Female & 446 & 54 & 379 & 54 & 825 & 54 & \\
\hline \multicolumn{8}{|l|}{$\mathrm{Age}^{\mathrm{a}}$} \\
\hline Mean (sd) & 12.2 & $(2.4)$ & 12.1 & $(2.0)$ & 12.2 & $(2.3)$ & 0.51 \\
\hline $8-11$ years & 352 & 42 & 303 & 43 & 655 & 42 & 0.83 \\
\hline $12-16$ years & 481 & 58 & 405 & 57 & 786 & 58 & \\
\hline
\end{tabular}

a baseline, we averaged the 1987 and 1989 data to permit analyses over 3-years (the mean of 1987/1989 followed to 1991) and 9-years (the mean of $1987 / 1989$ followed to 1997) intervals like those analyzed for the US sample. Ages were 8-16 years in 1987/1989, 11-20 years in 1991, and 17-26 years in 1997. Only Dutch children with complete data at all assessments were included in the analyses $(N=708$; see Table 1$)$. Dropout rates were higher for younger children, but did not differ by problem scores, gender, or socioeconomic status [11].

\section{Measures}

\section{Emotional and behavioral problems}

For the US sample, parents completed the AchenbachConners-Quay Behavior Checklist (ACQ; [4] in 1986, and the Child Behavior Checklist (CBCL; [1] at the 3-year assessment. For the Dutch sample, parents completed the CBCL at the baseline and subsequent assessments, until the 9-year assessment. At this assessment, parents in both samples completed the Young Adult Behavior Checklist (YABCL; [2]). These measures possess good discriminative validity [1], predictive validity [24], and cross-cultural validity [7].The rating forms include seven narrow-band syndromes that span from childhood into adulthood: Withdrawn, Anxious/Depressed, Somatic Complaints, Delinquent Behavior, Aggressive Behavior, Thought Problems, and Attention Problems (Table 2). To compare scores from the ACQ, CBCL, and YABCL, we converted raw syndrome scores to standard scores with a mean of 100 and a standard deviation of 15 within each assessment 
Table 2 Adjusted mean scores (standardized to mean =100) and standard errors by SES at baseline, 3-, and 9-year assessments, and significance of model parameters

\begin{tabular}{|c|c|c|c|c|c|c|c|c|}
\hline SES & $\begin{array}{l}\text { Time } \\
\text { (year) }\end{array}$ & Withdrawn & $\begin{array}{l}\text { Anxious/ } \\
\text { depressed }\end{array}$ & $\begin{array}{l}\text { Somatic } \\
\text { complaints }\end{array}$ & $\begin{array}{l}\text { Delinquent } \\
\text { behavior }\end{array}$ & $\begin{array}{l}\text { Aggressive } \\
\text { behavior }\end{array}$ & $\begin{array}{l}\text { Thought } \\
\text { problems }\end{array}$ & $\begin{array}{l}\text { Attention } \\
\text { problems }\end{array}$ \\
\hline Low & 0 & $100.92(0.66)$ & $100.56(0.65)$ & $100.06(0.63)$ & $99.80(0.58)$ & $101.33(0.64)$ & $99.66(0.54)$ & $100.88(0.65)$ \\
\hline Medium & 0 & $99.14(0.53)$ & $99.46(0.50)$ & $99.35(0.49)$ & $98.12(0.45)$ & $99.32(0.50)$ & $98.48(0.42)$ & $99.25(0.51)$ \\
\hline High & 0 & $99.63(0.80)$ & $99.98(0.79)$ & $99.41(0.76)$ & $97.93(0.71)$ & $98.42(0.78)$ & $97.35(0.66)$ & $97.71(0.79)$ \\
\hline Low & 3 & $100.77(0.68)$ & $100.08(0.69)$ & $100.20(0.69)$ & $99.91(0.64)$ & $99.37(0.64)$ & $99.38(0.60)$ & $99.53(0.66)$ \\
\hline Medium & 3 & $99.14(0.53)$ & $99.62(0.54)$ & $99.92(0.54)$ & $98.80(0.50)$ & $98.55(0.50)$ & $99.12(0.47)$ & $98.89(0.51)$ \\
\hline High & 3 & $100.52(0.82)$ & $100.01(0.84)$ & $100.26(0.84)$ & $98.09(0.77)$ & $97.25(0.78)$ & $97.93(0.73)$ & $97.99(0.80)$ \\
\hline Low & 9 & $100.54(0.65)$ & $100.86(0.69)$ & $100.49(0.66)$ & $99.22(0.68)$ & $100.96(0.67)$ & $100.50(0.67)$ & $100.64(0.70)$ \\
\hline Medium & 9 & $99.05(0.51)$ & $99.65(0.54)$ & $99.49(0.51)$ & $100.11(0.53)$ & $99.74(0.52)$ & $99.26(0.52)$ & $100.97(0.54)$ \\
\hline High & 9 & $96.91(0.79)$ & $98.70(0.84)$ & $97.99(0.80)$ & $99.95(0.82)$ & $97.45(0.82)$ & $96.94(0.81)$ & $98.34(0.85)$ \\
\hline \multicolumn{2}{|c|}{ Model parameters } & $P$ & $P$ & $P$ & $P$ & $P$ & $P$ & $P$ \\
\hline \multicolumn{2}{|l|}{ SES } & $0.02^{\mathrm{a}}$ & 0.37 & 0.37 & 0.39 & 0.003 & 0.002 & $0.02^{\mathrm{a}}$ \\
\hline \multicolumn{2}{|l|}{ Country } & 0.08 & 0.36 & 0.14 & 0.57 & $0.04^{\mathrm{a}}$ & 0.67 & 0.53 \\
\hline \multicolumn{2}{|l|}{ Sex } & 0.09 & 0.0006 & $<0.0001$ & $<0.0001$ & $<0.0001$ & 0.84 & $<0.0001$ \\
\hline \multicolumn{2}{|l|}{ Age } & 0.28 & $0.008^{\mathrm{a}}$ & 0.39 & 0.09 & $<0.0001$ & 0.07 & 0.0004 \\
\hline \multicolumn{2}{|c|}{ Time*SES } & 0.009 & 0.38 & 0.28 & 0.06 & 0.30 & 0.46 & 0.10 \\
\hline \multicolumn{2}{|c|}{ Country*SES } & 0.88 & 0.95 & 0.11 & 0.68 & 0.77 & 0.75 & 0.47 \\
\hline \multicolumn{2}{|c|}{ Country*time } & 0.02 & 0.10 & 0.13 & 0.71 & 0.42 & 0.64 & 0.66 \\
\hline \multicolumn{2}{|c|}{ Time* country*SES } & 0.96 & 0.77 & 0.01 & 0.23 & 0.46 & 0.41 & 0.82 \\
\hline
\end{tabular}

Unstructured covariance matrix; random intercept and random slope; three time points: baseline, after 3 and 9 years. Age in two groups: 8-11 and 12-16 at baseline. $N=1,541$, observations: 4,623. SES socioeconomic status

${ }^{a}$ When corrected for number of comparisons [20], these effects were most likely to be significant by chance

time. A case was defined as an individual who had a clinical elevation on a syndrome ( $\geq 1 \mathrm{SD}$ above the mean) at a particular assessment time. Cumulative prevalence was calculated from the baseline assessment to the 3- and 9 -year assessments.

\section{Socioeconomic status}

Socioeconomic status (SES) was coded according to the occupational level of the parents at baseline (i.e., 1986 for US sample and 1987/1989 for Dutch sample). In the US sample, parental occupation was coded according to Hollingshead's [12] 9-point occupational index for the parent having the higher occupational status. In the Dutch sample, parental occupation was first coded according to van Westerlaak [22] and recoded according to Hollinghead's occupational index. Parental occupation was divided into three groups low (1-4), medium (5-7), and high (8-9). Low occupations include among others unskilled and semi-skilled workers, and skilled manual workers; medium occupations include clerical and sales workers, technicians, semiprofessionals, minor professionals, and small business owners; high occupations include administrators, lesser professionals, owners of medium sized or large businesses, higher executives, and major professionals.

Statistical analyses

We used logistic regression and mixed linear regression models that included SES, country, age (dichotomized as 8-11 and 12-16 years at baseline) and gender. Interactions of SES with country were tested to identify country-specific associations of SES with the syndromes. First, to test differential rates of psychopathology over time for the three SES levels, data were analyzed with mixed linear models. We allowed the intercept and regression coefficients to differ randomly among participants. These models estimate group means with standard errors [21]. A significant main effect of SES indicated that syndrome scores differed by SES level. Significant interactions of country by SES indicated country-specific effects; significant interactions between time and SES indicated differential changes in syndrome scores over time by SES; and significant interactions of country $\times$ SES $\times$ time, indicated differential changes by SES that were country specific. Second, to test differential cumulative prevalence, we used logistic regression models with the cumulative prevalence 
over three assessments as dependent and SES at baseline as independent variables. We additionally ran the analyses for new cases during follow-up only, thus excluding individuals with clinical elevations on the syndromes at baseline. This yielded similar odds ratios to those for the cumulative prevalence rates presented in Table 3 .

\section{Results}

As Table 1 shows, the samples did not differ significantly by gender or age, but high SES was significantly more common in the US than the Dutch sample $(P=0.001)$. Associations between SES at baseline and subsequent

Table 3 Odds Ratios for proportions of participants who ever had clinically elevated scores, by SES

\begin{tabular}{|c|c|c|c|}
\hline Syndrome & Cases, $N(\%)$ & Odds ratios & $P^{\mathrm{a}}$ \\
\hline Withdrawn & & & 0.21 \\
\hline Low & $140(31)$ & $1.43(1.03-1.97)$ & \\
\hline Medium & $195(26)$ & $1.14(0.85-1.55)$ & \\
\hline High & $81(24)$ & 1.00 & \\
\hline Anxious/depressed & & & 0.75 \\
\hline Low & $139(30)$ & $1.21(0.88-1.65)$ & \\
\hline Medium & $204(27)$ & $1.04(0.77-1.39)$ & \\
\hline High & $90(27)$ & 1.00 & \\
\hline Somatic complaints & & & 0.18 \\
\hline Low & $125(27)$ & $0.94(0.68-1.29)$ & \\
\hline Medium & $186(25)$ & $0.83(0.62-1.12)$ & \\
\hline High & $98(30)$ & 1.00 & \\
\hline Delinquent behavior & & & 0.73 \\
\hline Low & $102(22)$ & $1.15(0.81-1.64)$ & \\
\hline Medium & $156(21)$ & $1.01(0.73-1.40)$ & \\
\hline High & $65(20)$ & 1.00 & \\
\hline Aggressive behavior & & & 0.57 \\
\hline Low & $135(29)$ & $1.81(1.28-2.55)$ & \\
\hline Medium & $194(26)$ & $1.45(1.04-2.00)$ & \\
\hline High & $62(19)$ & 1.00 & \\
\hline Thought problems & & & 0.25 \\
\hline Low & $120(26)$ & $2.03(1.41-2.93)$ & \\
\hline Medium & $164(22)$ & $1.60(1.13-2.26)$ & \\
\hline High & $53(16)$ & 1.00 & \\
\hline Attention problems & & & 0.03 \\
\hline Low & $134(29)$ & $1.40(1.01-1.96)^{b}$ & \\
\hline Medium & $223(30)$ & $1.39(1.02-1.89)^{b}$ & \\
\hline High & $76(23)$ & 1.00 & \\
\hline
\end{tabular}

Odds ratios and $95 \%$ confidence intervals are adjusted for country, gender and age. Significant odds ratios $(P<0.05)$ are in boldface

${ }^{a} P$ value for interaction of SES with country

b When corrected for number of comparisons [20], these effects were most likely to be significant by chance syndrome scores are presented in Table 2. Low SES significantly predicted high Withdrawn, Aggressive Behavior, Thought Problems, and Attention Problems scores in both the US and Dutch samples, with no significant interactions between SES and country. The association with SES did not change over time for Aggressive Behavior, Thought Problems, or Attention Problems. For Withdrawn, the effect of SES decreased from baseline to the 3-year assessment, but then increased again at the 9-year assessment. The only country-specific finding was for Somatic Complaints: In the Dutch sample, there were no significant SES effects, whereas in the US sample significant SES differences were found in the 3- and 9-year assessments. No interactions of SES with gender or age were significant.

Table 3 displays odds ratios and proportions of participants who ever had clinically elevated syndrome scores at any of the three assessments. As Table 3 shows, significantly greater proportions of lower SES than higher SES participants had elevated scores on the following syndromes: Withdrawn, Aggressive Behavior, Thought Problems, and Attention Problems. This may reflect both differential incidence and worse recovery rates. The only difference between the US and The Netherlands was for Attention Problems, where in The Netherlands the cumulative prevalence rate was elevated in both the low and the medium SES groups, versus only in the US low SES group. Analyses of cases that became deviant only at the 3- or 9-year assessments yielded odds ratios like those for the cumulative prevalence rates shown in Table 3.

\section{Discussion}

This study was designed to test the extent to which previous findings on the association between SES and emotional/behavioral problems in the United States can be generalized to The Netherlands. We found very few crossnational differences. For both countries, SES predicted syndrome scores and cumulative prevalence rates of elevated scores for withdrawn and aggressive behavior, as well as for thought and attention problems. The SES gradient in syndrome scores was generally stable over the ages that were studied, except that SES differences for the withdrawn syndrome increased in young adulthood. Between countries, the main significant differences were for somatic complaints, where an SES effect was found only in the US sample, and for the cumulative prevalence of deviant scores on attention problems, where the SES gradient was stronger in the Dutch sample. The crossnational difference for attention problems was most likely to be significant by chance when corrected for number of comparisons. The finding for somatic complaints is in line with our hypothesis that we expected a stronger SES 
gradient in the US than in the Netherlands based on the latter country's free access to health care and a high intensity of monitoring and identification of mental health problems in primary and secondary schools.

Our findings argue for social causation effects on a variety of syndromes. The findings support a diathesisstress model of psychopathology whereby factors associated with low parental occupation or status may raise risks for emotional/behavioral problems. Low parental occupation or status may be a proxy for other factors, such as parental conflict, and parenting styles, but also more material factors such as the safety of the neighborhood, type of health insurance, and poverty [10, 18]. Our results do not exclude selection effects, though, as it is possible that mental health problems of parents may affect their occupational status and eventually their SES [13, 17, 19], which in turn affects the mental health of their offspring.

Our findings were very similar to those of Wadsworth and Achenbach [25] for their US sample. Wadsworth and Achenbach found more interactions of SES with time, indicating increasing socioeconomic differences for somatic complaints, aggressive behavior, delinquent behavior, thought problems, and attention problems. A difference between the previous and current study is that Wadsworth and Achenbach [25] used a combination of occupation, income, and receipt of federal assistance to measure SES, whereas we used only occupation. Additional analyses of the US sample yielded stronger associations with emotional and behavioral problems for the income-based variables than for occupation. Differences between countries might be greater for income-based than occupational indicators of SES, as income level is a predictor of access to mental health care in some but not all countries [26].

The combination of the US and Dutch samples offered a unique framework for comparing associations of SES with emotional and behavioral problems prospectively between countries. Longitudinal studies of SES and mental health are rare, and direct cross-national comparisons are even rarer $[6,8]$. The Dutch and US samples were comparable in many ways, such as being assessed with the same instrument in the mid-1980s through mid-1990s, being population based, and covering the same age ranges. The samples did differ, though, as the US sample was a nationally representative sample, whereas the Dutch sample was representative of one of the provinces of the Netherlands, including both densely populated and rural areas. Furthermore, the Dutch occupational codes [22] were translated into Hollingshead's codes. The coding systems are very similar, and the correlation between scores was high (0.87). Some other limitations apply to the present study. To ensure continuity across assessment periods, emotional and behavioral problems were measured with parent checklists only. Further studies using direct assessments through diagnostic interviews should extend the results by testing the extent to which the findings can be generalized to youths with psychiatric disorders. Socioeconomic status may be optimally measured with variables including income, occupation, and education. However, only occupation was available for the Dutch sample. Other indicators of SES might have yielded different results. For example, family income might have different relations to the developmental course of emotional/behavioral problems. Unfortunately, information on utilization of mental health services was not collected systematically for the Dutch sample. Although we did find SES differences in emotional and behavioral problems, the differences were small. This does not mean they are of no importance, as small differences can have considerable effects at the population level. Furthermore, SES is only one of the many factors associated with psychopathology in children and young adults.

In conclusion, in both analyses of rates and of cumulative prevalence, low SES was associated with more emotional and behavioral problems in childhood, adolescence, and young adulthood. The SES gradient was similar for the US and Dutch populations and remained stable over the development from childhood to young adulthood. As SES differences did not decrease through the 9-year assessment, it remains important to address these differences through prevention with special attention to lower SES groups.

Open Access This article is distributed under the terms of the Creative Commons Attribution Noncommercial License which permits any noncommercial use, distribution, and reproduction in any medium, provided the original author(s) and source are credited.

\section{References}

1. Achenbach TM (1991) Manual for the Child Behavior Checklist 4-18 and 1991 Profiles. University of Vermont, Department of Psychiatry, Burlington

2. Achenbach TM (1997) Manual for the Young Adult Self-Report and Young Adult Behavior Checklist. University of Vermont, Department of Psychiatry, Burlington

3. Achenbach TM, Howell CT, McConaughy SH, Stanger C (1995) Six-year predictors of problems in a national sample: III. Transitions to young adult syndromes. J Am Acad Child Adolesc Psychiatry 34:658-669

4. Achenbach TM, Howell CT, Quay HC, Conners CK (1991) National survey of problems and competencies among four- to sixteen-year-olds: parents' reports for normative and clinical samples. Monogr Soc Res Child Dev 56:1-131

5. Alegria M, Bijl RV, Lin E, Walters EE, Kessler RC (2000) Income differences in persons seeking outpatient treatment for mental disorders: a comparison of the United States with Ontario and The Netherlands. Arch Gen Psychiatry 57:383-391

6. Ashford J, Smit F, van Lier PA, Cuijpers P, Koot HM (2008) Early risk indicators of internalizing problems in late childhood: a 9-year longitudinal study. J Child Psychol Psychiatry 49:774780 
7. de Groot A, Koot HM, Verhulst FC (1994) Cross-cultural generalizability of the Child Behavior Checklist cross-informant syndromes. Psychol Assess 6:225-230

8. Fergusson DM, Horwood LJ, Lawton JM (1990) Vulnerability to childhood problems and family social background. J Child Psychol Psychiatry 31:1145-1160

9. Fryers T, Melzer D, Jenkins R (2003) Social inequalities and the common mental disorders: a systematic review of the evidence. Soc Psychiatry Psychiatr Epidemiol 38:229-237

10. Grant KE, Compas BE, Stuhlmacher AF, Thurm AE, McMahon SD, Halpert JA (2003) Stressors and child and adolescent psychopathology: moving from markers to mechanisms of risk. Psychol Bull 129:447-466

11. Hofstra MB, Van Der Ende J, Verhulst FC (2001) Adolescents' self-reported problems as predictors of psychopathology in adulthood: 10-year follow-up study. Br J Psychiatry 179:203-209

12. Hollingshead AB (1975) Four-factor index of social-status. Yale University, New Haven

13. Huurre T, Rahkonen O, Komulainen E, Aro H (2005) Socioeconomic status as a cause and consequence of psychosomatic symptoms from adolescence to adulthood. Soc Psychiatry Psychiatr Epidemiol 40:580-587

14. Johnson JG, Cohen P, Dohrenwend BP, Link BG, Brook JS (1999) A longitudinal investigation of social causation and social selection processes involved in the association between socioeconomic status and psychiatric disorders. J Abnorm Psychol 108:490-499

15. Kuo AA, Inkelas M, Lotstein DS, Samson KM, Schor EL, Halfon N (2006) Rethinking well-child care in the United States: an international comparison. Pediatrics 118:1692-1702

16. Lorant V, Deliege D, Eaton W, Robert A, Philippot P, Ansseau M (2003) Socioeconomic inequalities in depression: a meta-analysis. Am J Epidemiol 157:98-112
17. McLeod JD, Kaiser K (2004) Childhood emotional and behavioral problems and educational attainment. Am Sociol Rev 69:636-658

18. McLoyd VC (1998) Socioeconomic disadvantage and child development. Am Psychol 53:185-204

19. Power C, Manor O (1992) Explaining social class differences in psychological health among young adults: a longitudinal perspective. Soc Psychiatry Psychiatr Epidemiol 27:284-291

20. Sakoda JM, Cohen BH, Beall G (1954) Test of significance for a series of statistical tests. Psychol Bull 51:172-175

21. Twisk JWR (2003) Applied longitudinal data analysis for epidemiology: a practical guide. Cambridge University Press, Cambridge

22. van Westerlaak JH, Kropman JH, Collaris JWM (1975) Manual for occupational level [Beroepenklapper]. Institute for Sociology, Nijmegen

23. Verhulst FC, Akkerhuis GW, Althaus M (1985) Mental health in Dutch children: (I). A cross-cultural comparison. Acta Psychiatr Scand Suppl 323:1-108

24. Verhulst FC, Koot HM, Van der Ende J (1994) Differential predictive value of parents' and teachers' reports of children's problem behaviors: a longitudinal study. J Abnorm Child Psychol 22:531-546

25. Wadsworth ME, Achenbach TM (2005) Explaining the link between low socioeconomic status and psychopathology: testing two mechanisms of the social causation hypothesis. J Consult Clin Psychol 73:1146-1153

26. Zwaanswijk M, Verhaak PF, Bensing JM, van der Ende J, Verhulst FC (2003) Help seeking for emotional and behavioural problems in children and adolescents: a review of recent literature. Eur Child Adolesc Psychiatry 12:153-161 